OPEN ACCESS

Edited by:

Manfred Eggersdorfer

DSM, Switzerland

Reviewed by:

Yoshitaka Hashimoto,

Kyoto Prefectural University of

Medicine, Japan

Moncef Feki,

Tunis El Manar University, Tunisia

*Correspondence:

Long Zhou

wcuzhl@163.com

Gang Li

ligang8252@qq.com

†These authors share first authorship

Specialty section:

This article was submitted to

Clinical Nutrition

a section of the journa

Frontiers in Nutrition

Received: 06 January 2022 Accepted: 07 February 2022

Published: 25 February 2022

Citation:

Zhou L, Huang H, Wen X, Chen Y,

Liao J, Chen F, Zhao L, Liu M, Tao J

and Li G (2022) Associations of Serum

and Red Blood Cell Folate With

All-Cause and Cardiovascular

Mortality Among Hypertensive

Patients With Elevated Homocysteine.

Front. Nutr. 9:849561.

doi: 10.3389/fnut.2022.849561

\section{Associations of Serum and Red Blood Cell Folate With All-Cause and Cardiovascular Mortality Among Hypertensive Patients With Elevated Homocysteine}

\author{
Long Zhou ${ }^{1 * \dagger}$, Hui Huang ${ }^{1 \dagger}$, Xiaoxiao Wen ${ }^{2}$, Yu Chen ${ }^{1}$, Jie Liao ${ }^{1}$, Fuli Chen ${ }^{1}$, \\ Liancheng Zhao ${ }^{3}$, Mingjiang Liu ${ }^{1}$, Jianhong Tao ${ }^{1}$ and Gang $\mathrm{Li}^{1 *}$ \\ ${ }^{1}$ Department of Cardiology, Sichuan Provincial People's Hospital, University of Electronic Science and Technology of China, \\ Chengdu, China, ${ }^{2}$ Department of Epidemiology, College of Public Health and Health Professions and College of Medicine, \\ University of Florida, Gainesville, FL, United States, ${ }^{3}$ Division of Prevention and Community Health, National Center for \\ Cardiovascular Disease, Fuwai Hospital, Chinese Academy of Medical Sciences and Peking Union Medical College, \\ Beijing, China
}

Objectives: This study aims to explore the associations between serum and red blood cell (RBC) folate as indicators of short- and long-term folate status, respectively, and allcause as well as CVD mortality among hypertensive patients with elevated homocysteine.

Methods: A prospective cohort study of the National Health and Nutrition Examination Survey (1999-2006) and 2015 Linked Mortality File was performed. All-cause and CVD mortality risk estimated using Cox proportional hazards models with adjusting for multiple potential covariates.

Results: A total of 1,753 hypertensive patients with elevated homocysteine [mean (SD) age, 68.5 (13.1)] were included in the analysis. During a median follow-up of 10.0 years, a total of 899 all-cause and 257 CVD deaths occurred. Compared the highest with the lowest quartile of RBC folate, the multivariable adjusted hazard ratios and 95\% confidence intervals for all-cause and CVD death were 1.13 (0.92-1.39) and 1.47 (1.01-2.16) respectively. There was a significant and positive trend between RBC folate and the risk of CVD death ( $p$ for trend $=0.0196$ ). No significant association was found between serum folate and mortality risk among the study sample.

Conclusions: High level of RBC folate is associated with an increased risk of cardiovascular mortality among hypertensive patients with elevated homocysteine while serum folate has no such effects.

Keywords: serum folate, red blood cell, hypertension, homocysteine, mortality

\section{INTRODUCTION}

Hypertension or high blood pressure (BP) is one of the leading modifiable risk factors for cardiovascular disease (CVD) and all-cause mortality worldwide $(1,2)$. It was estimated that the number of people aged 30-79 years with hypertension doubled from 1990 to 2019, from 331 million women and 317 million men in 1990 to 626 million women and 652 million men in 2019 (3). 
Both genetic and prospective cohort studies yield strong evidence that the association between homocysteine and CVD is causal, wherein lowering serum homocysteine concentrations by 3 $\mu \mathrm{mol} / \mathrm{L}$ was shown to reduce the risk of ischemic heart disease by $16 \%$ and stroke by $24 \%$ (4). More importantly, hypertension and elevated homocysteine levels may enhance the ability to provoke the risk of CVD and all-cause mortality each other (5). Previous studies showed that subjects with both elevated homocysteine $(\geq 10 \mu \mathrm{mol} / \mathrm{L})$ and hypertension (referred to as "H-type hypertension") have a significantly higher risk of stroke compared with those without either condition $(6,7)$. Folate is naturally present in a wide variety of foods, including vegetables (especially dark green leafy vegetables), fruits and fruit juices, nuts, beans, peas, seafood, eggs, dairy products, meat, poultry, and grains. Spinach, liver, asparagus, and brussels sprouts are among the foods with the highest folate levels (8). Folate is critically involved in homocysteine metabolism. Elevated homocysteine is usually closely linked to inadequate folate intake or status $(7,9)$. For these reasons, folic acid supplementation has been recommended for hypertensive patients, especially those with elevated homocysteine, as a primary prevention strategy of stroke (10). However, our previous analysis showed that the use of folic acid supplements could significantly increase red blood cell (RBC) folate concentrations, which were further associated with an increased risk of severe abdominal aortic calcification (11). In contrast to serum folate which reflects recent intakes, $\mathrm{RBC}$ folate reflects both the folate stored in the liver and the longterm average consumption of folate over the life span of RBCs (12). As an extension to our previous work in identifying the potential adverse health outcomes of high folate levels, this study aimed to determine the associations of serum and RBC folate with all-cause and cardiovascular mortality among hypertensive patients with elevated homocysteine using the National Health and Nutrition Examination Survey (NHANES), 1999-2006 and linked mortality data.

\section{METHODS}

\section{Study Population}

Data were derived from 4 cycles of the NHANES (19992000, 2001-2002, 2003-2004, and 2005-2006) surveys, which were administered by the National Center for Health Statistics (NCHS). A detailed description of the NHANES study design and methods are available elsewhere (13). Briefly, NHANES is an ongoing health-related program conducted with a complex, multistage, probability sampling design for a representative sample of US residents. For this study, we merged the NHANES 1999-2006 with the 2015 NCHS Linked Mortality Files. In total, 41,474 individuals completed the NHANES surveys between 1999-2006 within 4 cycles (9,965 in the NHANES 1,999-2,000, 11,039 in the NHANES 2001-2002, 10,122 in the NHANES 2003-2004, and 10,348 in the NHANES 2005-2006). The present study was limited to hypertensive patients with elevated homocysteine aged $\geq 20$ years with complete data on mortality and covariates, resulting in a sample of 1,753 participants in the analysis. A detailed flowchart depicting participant selection is shown in Figure 1. The NCHS institutional review board

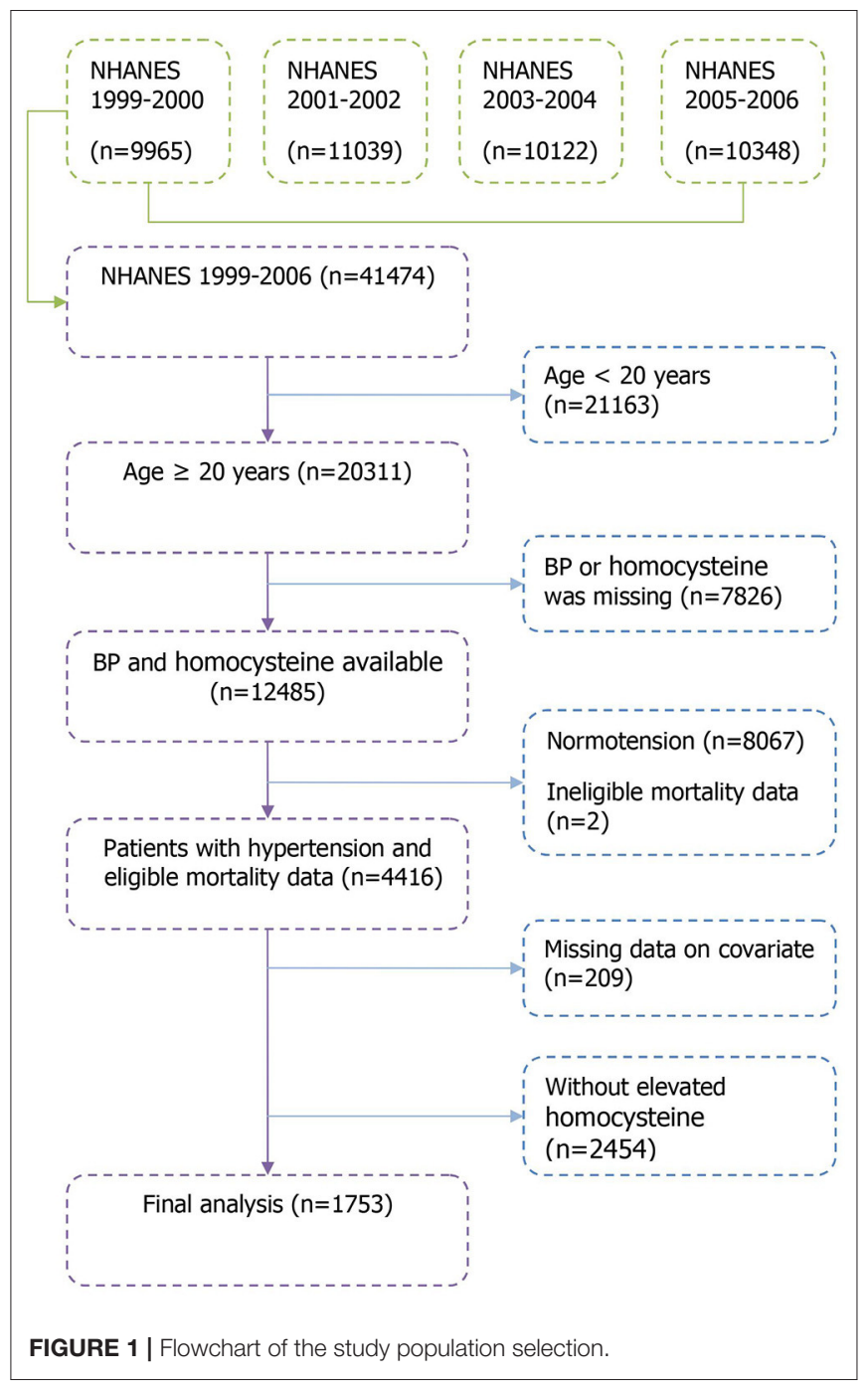

approved the study protocol and all participants provided written informed consent.

\section{Data Collection}

Data were collected through interviews, medical examination and subsequent laboratory assessments in the Mobile Examination Centre. The NHANES interviews were completed by trained interviewers in participants' homes by using a computer-assisted personal interview system.

Demographics, health conditions, and lifestyle information were obtained through questionnaires. Smoking status was categorized into current, former and never smoker according to participants' answers. Drinking status includes drinkers and nondrinkers, where participants who drank alcohol at least 12 times in the previous year were defined as drinkers.

Bodyweight $(\mathrm{kg})$ was measured using a digital weighing scale, and standing height $(\mathrm{cm})$ was measured using a stadiometer. Body mass index (BMI) was calculated using the formula: weight $(\mathrm{kg}) /[\text { height }(\mathrm{m})]^{2}$. BMI $\geq 25 \mathrm{~kg} / \mathrm{m}^{2}$ was defined as overweight/obese (14). For BP measurement, participants were 
TABLE 1 | Baseline characteristics of hypertensive patients with elevated homocysteine by quartiles of serum folate.

\begin{tabular}{|c|c|c|c|c|c|}
\hline Characteristics & Quartile 1 & Quartile 2 & Quartile 3 & Quartile 4 & $P$-values \\
\hline N & 429 & 447 & 434 & 443 & \\
\hline Age, y & $61.8 \pm 14.4$ & $67.7 \pm 12.5$ & $69.8 \pm 12.1$ & $74.5 \pm 9.6$ & $<0.0001$ \\
\hline Men, No. (\%) & $286(66.7)$ & $256(57.3)$ & $255(58.8)$ & $227(51.2)$ & $<0.0001$ \\
\hline Ethnicity, No. (\%) & & & & & $<0.0001$ \\
\hline Hispanic & $89(20.8)$ & $95(21.3)$ & $84(19.4)$ & $55(12.4)$ & \\
\hline White & $176(41.0)$ & 219 (49.0) & $263(60.6)$ & $331(74.7)$ & \\
\hline Black & $148(34.5)$ & 119 (26.6) & $78(18.0)$ & 45 (10.2) & \\
\hline Asian or others & $16(3.7)$ & $14(3.1)$ & $9(2.1)$ & $12(2.7)$ & \\
\hline Education, No. (\%) & & & & & 0.0046 \\
\hline Less than high school & $182(42.4)$ & 204 (45.6) & $175(40.3)$ & $142(32.1)$ & \\
\hline High school & $109(25.4)$ & 107 (23.9) & 118 (27.2) & $134(30.3)$ & \\
\hline More than high school & $138(32.2)$ & $136(30.4)$ & $141(32.5)$ & $167(37.7)$ & \\
\hline Smoking, No. (\%) & & & & & $<0.0001$ \\
\hline Never & $159(37.1)$ & $195(43.6)$ & $195(44.9)$ & $210(47.4)$ & \\
\hline Current & $130(30.3)$ & $88(19.7)$ & $65(15.0)$ & $27(6.1)$ & \\
\hline Former & $140(32.6)$ & $164(36.7)$ & $174(40.1)$ & 206 (46.5) & \\
\hline Drinker, No. (\%) & $279(65.0)$ & $274(61.3)$ & $276(63.6)$ & $254(57.3)$ & 0.0988 \\
\hline Overweight/obese, No. (\%) & 340 (79.3) & $345(77.2)$ & $324(74.7)$ & $315(71.1)$ & 0.0328 \\
\hline Diabetes, No. (\%) & $69(16.1)$ & $124(27.7)$ & $98(22.6)$ & $100(22.6)$ & 0.0006 \\
\hline Hypercholesterolemia, No. (\%) & $153(35.7)$ & $197(44.1)$ & $187(43.1)$ & $222(50.1)$ & 0.0003 \\
\hline Antihypertensive medication use, No. (\%) & $268(62.5)$ & $321(71.8)$ & $310(71.4)$ & $342(77.2)$ & $<0.0001$ \\
\hline eGFR, mL/min/1.73 m² & $77.3 \pm 23.8$ & $70.3 \pm 23.2$ & $68.9 \pm 21.8$ & $59.3 \pm 22.6$ & $<0.0001$ \\
\hline Log-transformed serum vitamin $B_{12}, p g / m L$ & $5.9 \pm 0.5$ & $5.9 \pm 0.5$ & $6.0 \pm 0.5$ & $6.2 \pm 0.6$ & $<0.0001$ \\
\hline Log-transformed CRP, mg/dL & $-1.1 \pm 1.2$ & $-1.2 \pm 1.2$ & $-1.4 \pm 1.1$ & $-1.3 \pm 1.1$ & 0.0009 \\
\hline
\end{tabular}

CRP, C-reactive protein; eGFR, estimated glomerular filtration rate. The cut-off values of serum folate were 8.3, 12.0, and 18.4 ng/mL.

asked to sit in a height-adjustable office-style chair and rest quietly for at least 5 min before taking BP readings. The mean of three systolic and diastolic BP readings was used in the analysis. Hypertension was defined as a systolic BP $\geq 140$ $\mathrm{mmHg}$ or a diastolic $\mathrm{BP} \geq 90 \mathrm{mmHg}$, and/or current use of antihypertensive medication.

\section{Laboratory Tests and Clinical Definitions}

Serum specimens were processed, stored, and shipped to the Division of Environmental Health Laboratory Sciences, National Center for Environmental Health, Centers for Disease Control and Prevention for analysis. Both serum folate and vitamin $B_{12}$ were measured by using the Bio-Rad Laboratories "Quantaphase II Folate/Vitamin $\mathrm{B}_{12}$ " radioassay kit. RBC folate was calculated from the whole blood folate concentration using microbiologic assay by adjusting for RBC volume and correcting for serum total folate concentration, which was calculated as the sum of individual folate forms. The formula to calculate RBC folate is as follows:

$$
\begin{array}{r}
\text { Red blood cell folate }=\{\text { whole blood folate }-[\text { serum blood } \\
\text { folate } \left.\left.^{*}(1.0-\text { hematocrit } / 100)\right]\right\} /(\text { hematocrit } / 100)
\end{array}
$$

Hemoglobin A1c (HbA1c) was measured by high-performance liquid chromatography (HPLC), and we defined diabetes as HbA1c $\geq 6.5 \%$ and/or current treatment with a hypoglycemic agent or insulin. The laboratory method used for total cholesterol (TC) measurement was enzymatic assay. We defined hypercholesterolemia as the presence of $\mathrm{TC} \geq 6.2 \mathrm{mmol} / \mathrm{L}$ or current medication use. C-reactive protein (CRP) was measured by latex-enhanced nephelometry on a Behring Nephelometer. The Chronic Kidney Disease Epidemiology Collaboration equation was used to estimate glomerular filtration rate (eGFR, in $\mathrm{mL} / \mathrm{min} / 1.73 \mathrm{~m}^{2}$ ) (15). Total homocysteine in plasma was measured by the Abbott Homocysteine assay on the Abbott AxSym analyzer, a fully automated fluorescence polarization immunoassay (FPIA) method.

\section{Ascertainment of Mortality Outcomes}

Mortality information was extracted from the 2015 NCHS Public-Use Linked Mortality Files, including mortality status, cause of death, and follow-up time of all included participants from the date of survey participation until death or December 31,2015 , whichever was earlier. The leading cause of death falls into 9 cause-specific death categories based on the International Classification of Diseases, 10th Revision (ICD-10) code on the participant's death certificate (16). We defined all-cause mortality as death because of any reason, while cardiovascular mortality included deaths reportedly due to disease of the heart (leading death code 001) or cerebrovascular diseases (leading death code 005) (17). 


\section{Statistical Analysis}

Data were presented as mean \pm standard deviation (SD) for continuous variables and as frequency (percentage) for categorical variables. RBC folate level was used in the analysis both as a categorical (quartiles) and a continuous (log-transformed) variable. We tested differences in baseline characteristics among folate quartile groups with one-way analysis of variance for continuous variables and $\chi 2$ tests for categorical variables. Crude all-cause and cardiovascular mortality rates by baseline serum and RBC folate quartiles were analyzed with the Kaplan-Meier curves and compared with the log-rank test. We then used Cox proportional hazards models to determine the hazard ratios (HRs) and 95\% confidence intervals (CIs) for the risks of all-cause and cardiovascular mortality in each serum and RBC folate quartiles, using the lowest quartile as the reference. The models were adjusted for several potential confounding variables including age, sex, ethnicity, education, smoking status, drinking, overweight/obesity, diabetes, hypercholesterolemia, antihypertensive medication use, eGFR, serum vitamin $B_{12}$, and CRP. To test for trend, the median value of each serum and $\mathrm{RBC}$ folate quartiles were used as continuous variables in the models. A two-tailed $p$ value $<0.05$ was considered statistically significant.

\section{RESULTS}

A total of 1,753 hypertensive patients with elevated homocysteine (serum homocysteine $\geq 10 \mu \mathrm{mol} / \mathrm{L}$ ) were included in the analysis. The mean (SD) age was 68.5 (13.1) years at baseline. The baseline characteristics of the study sample by serum and RBC folate quartiles are shown in Tables 1, 2. Overall, patients who had higher folate levels were more likely to be older, women, white, of higher education levels and to have hypercholesterolemia. Besides, patients with higher folate levels were more likely to have higher serum vitamin $B_{12}$ and lower eGFR levels.

During a median follow-up of 10.0 years, a total of 899 allcause deaths including 257 CVD deaths occurred among the 1,753 hypertensive patients with elevated homocysteine. The Kaplan-Meier curves indicate a graded increased risk of all-cause and CVD mortality associated with higher serum and RBC folate concentrations (log-rank $p<0.05)$ (Figures 2, 3).

The unadjusted and adjusted HRs and 95\% CIs of serum and $\mathrm{RBC}$ folate for all-cause and CVD mortality are shown in Table 3. Comparison of the highest with the lowest quartile of serum folate showed a higher risk of all-cause (HR: 1.83, 95\% CI: $1.52-2.20$ ) and CVD (HR: 1.61, 95\% CI: 1.14-2.29) mortality in the unadjusted model. However, the associations were no longer significant after adjustment for age, sex, ethnicity,

TABLE 2 | Baseline characteristics of hypertensive patients with elevated homocysteine by quartiles of red blood cell folate.

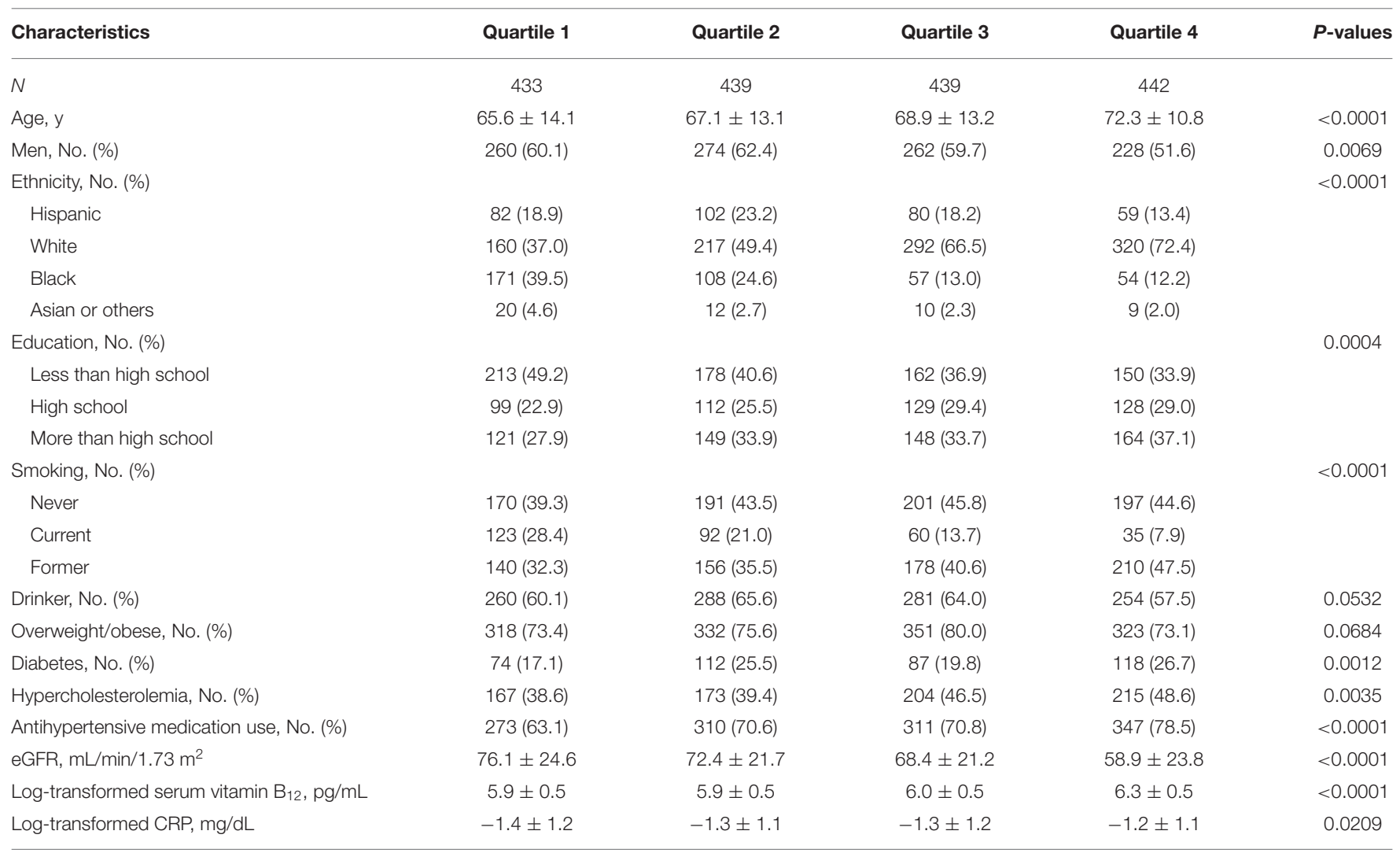

CRP, C-reactive protein; eGFR, estimated glomerular filtration rate. The cut-off values of RBC folate were 209, 278, and $381 \mathrm{ng} / \mathrm{mL}$. 


\section{All-cause death}

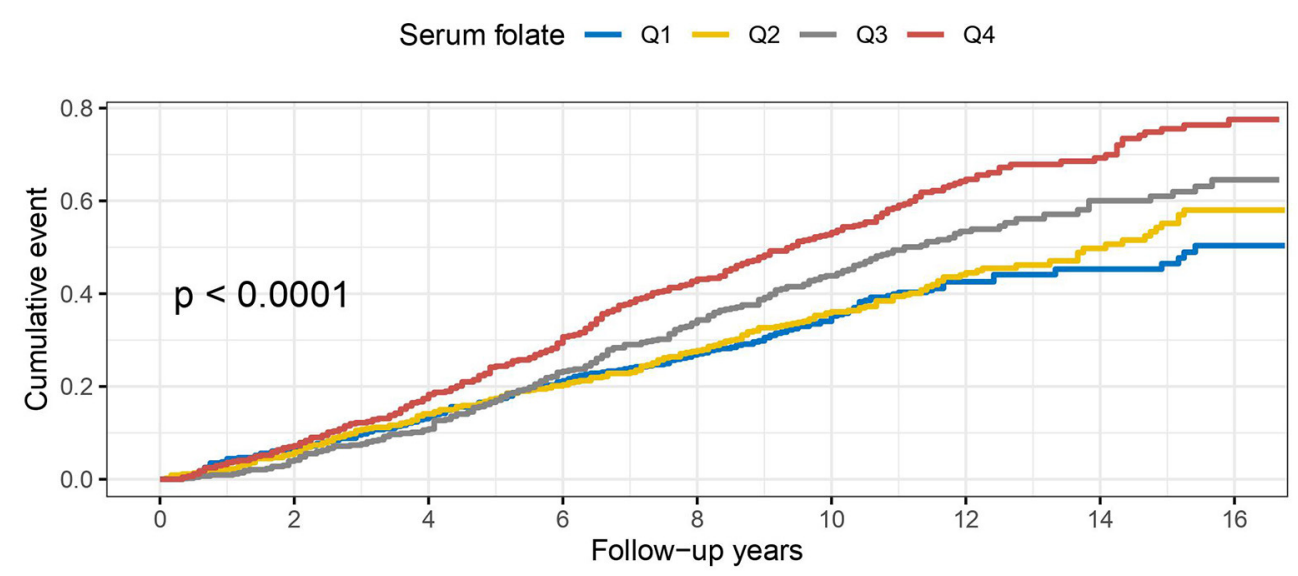

CVD death

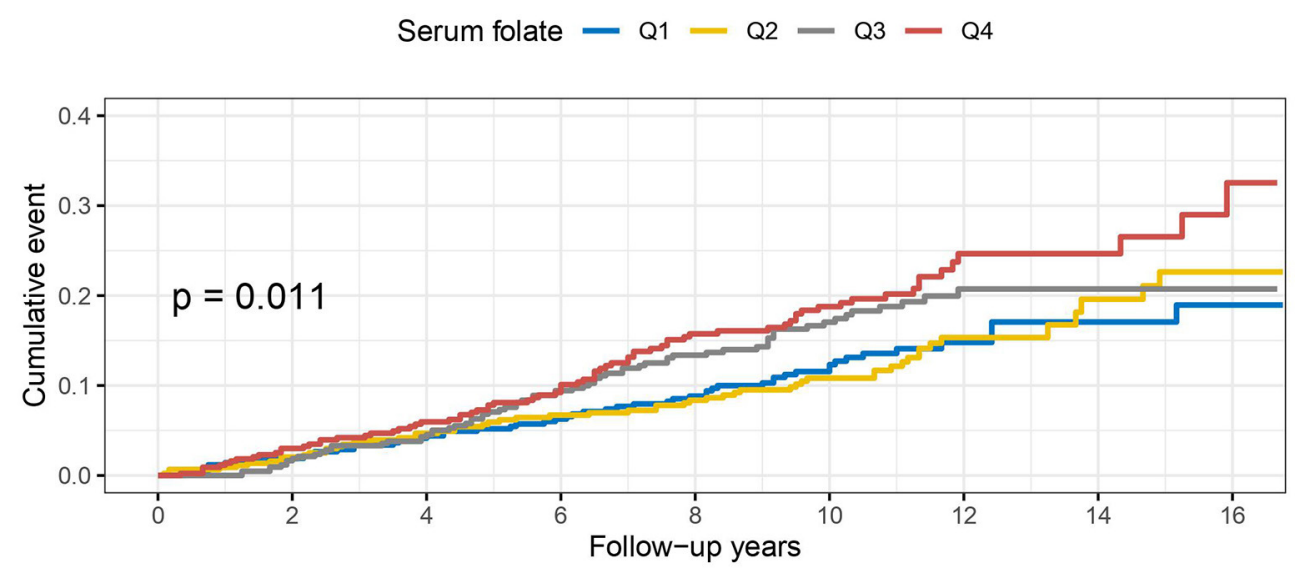

FIGURE 2 | Kaplan-Meier curves of all-cause and cardiovascular disease (CVD) death by baseline quartiles of serum folate.

education, smoking status, drinking, overweight/obesity, diabetes, hypercholesterolemia, antihypertensive medication use, eGFR, serum vitamin $\mathrm{B}_{12}$, and CRP. For RBC folate, the unadjusted HRs and 95\% CIs comparing the highest to lowest quartile were 1.64 (1.36-1.97) for all-cause death and 1.91 (1.352.70) for CVD death. After further adjustments for covariates, the HRs and 95\% CIs were $1.13(0.92-1.39)$ for all-cause death and 1.47 (1.01-2.16) for CVD death. There was a significant and positive trend between RBC folate and the risk of CVD death among hypertensive patients with elevated homocysteine ( $p$ for trend $=0.0196)$.

\section{DISCUSSION}

In this prospective cohort study of a nationally representative sample of US adults, we found that RBC folate was positively associated with the risk of cardiovascular mortality among patients with both hypertension and elevated homocysteine, which is referred to as H-type hypertension. This association was independent of multiple potential covariates including demographics, lifestyles, cardiometabolic risk factors, renal function, and others. However, no significant association was found between serum folate and mortality after adjusting for potential covariates.

Although the associations between folate levels and mortality have been previously investigated, the current evidence is not yet conclusive: some studies did not find any significant associations (18, 19); among studies that did, J- or U-shaped associations (20-23), positive linear associations (24), and inverse associations $(17,25,26)$ have all been reported; additionally, two studies found that only low folate levels were significantly associated with increased risk of mortality $(27,28)$. However, most of the previous studies used serum folate as the main exposure, and only two studies used RBC folate in the analysis $(21,24)$. This might be an explanation for the conflicting results, as serum folate only reflects recent intakes while $\mathrm{RBC}$ folate reflects long-term average consumption of folate (12). 


\section{All-cause death}

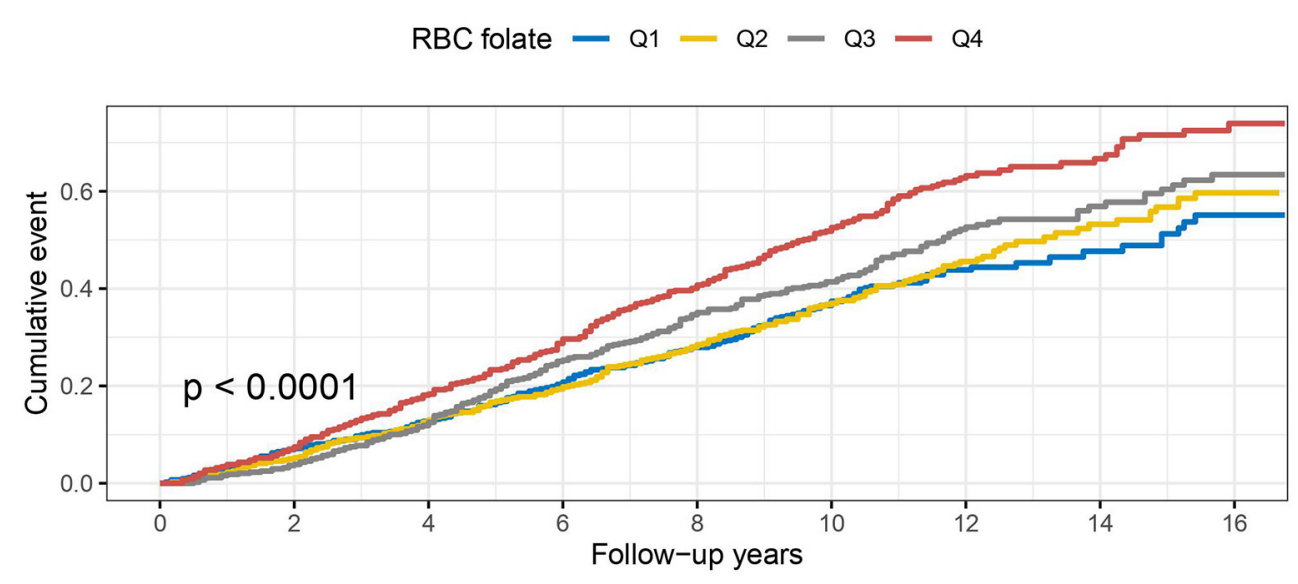

CVD death

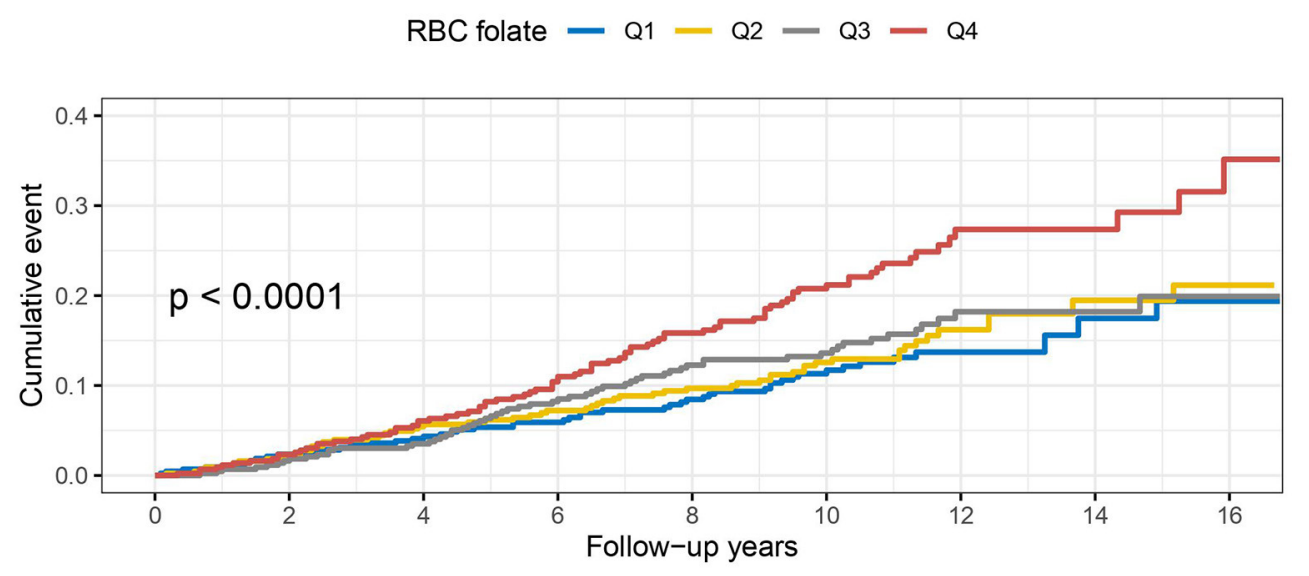

FIGURE 3 | Kaplan-Meier curves of all-cause and cardiovascular disease (CVD) death by baseline quartiles of red blood cell (RBC) folate.

To our knowledge, no previous studies specifically explored the long-term effects of serum and RBC folate on the risk of mortality among hypertensive patients with elevated homocysteine. Folate plays an important role in the metabolism of homocysteine by serving as the methyl donor in the conversion of homocysteine to methionine (29). Folic acid supplementation has been observed to be effective in reducing plasma homocysteine levels (30). Folic acid therapy has been recommended to patients with "H-type hypertension" to achieve potential cardiovascular benefits $(7,10)$. Our previous analysis found that the RBC folate levels in folic acid supplement users were much higher than those in non-users (mean RBC folate concentration: 728 vs. $494 \mathrm{ng} / \mathrm{mL}$ ) (11). The current study suggests that long-term cardiovascular death risk should be assessed properly when folic acid therapy is considered for patients diagnosed with H-type hypertension.

Considering that older people are at greater risk of death than younger adults. In addition to adjusting for age, we also performed stratified analysis according to age $(\leq 65$ or $>65$ years). The adjusted HRs of per 1-unit increase of logtransformed RBC folate for CVD death in younger and older adults were $1.24(0.62-2.44)$ and $1.43(1.03-1.99)$, respectively. However, no significant interaction was found between RBC folate and age ( $\mathrm{p}$ for interaction $=0.4592$ ), which means that the relationship between $\mathrm{RBC}$ folate level and CVD death in different age groups cannot be considered to have essential difference.

The mechanism underlying the association between high RBC folate and increased risk of CVD mortality among hypertensive patients with elevated homocysteine remains unclear. One possible explanation is homocysteine-induced arterial calcification and atherosclerosis (31). As mentioned above, although folate serves as the methyl donor in the conversion of homocysteine to methionine, high folate status may decrease the availability of the methyl donor or impair the activity of methyl-THF reductase, which is responsible for the methylation of homocysteine (32). Consequently, 
TABLE 3 | Mortality risk among hypertensive patients with elevated homocysteine by quartiles of serum and red blood cell folate.

\begin{tabular}{|c|c|c|c|c|c|}
\hline & Quartile 1 & Quartile 2 & Quartile 3 & Quartile 4 & $P$ for trend \\
\hline \multicolumn{6}{|l|}{ Serum folate } \\
\hline Person-years of follow-up & 4,066 & 4,294 & 4,001 & 3,777 & \\
\hline \multicolumn{6}{|l|}{ All-cause mortality } \\
\hline No. with events & 177 & 200 & 230 & 292 & \\
\hline Mortality rate, per 1,000 person-years & 43.5 & 46.6 & 57.5 & 77.3 & \\
\hline Adjusted HR $(95 \% \mathrm{Cl})^{\mathrm{a}}$ of per 1-unit increase of log-transformed folate & \multicolumn{4}{|c|}{$1.03(0.91-1.16)$} & 0.6457 \\
\hline \multicolumn{6}{|l|}{ Cardiovascular mortality } \\
\hline No. with events & 53 & 56 & 70 & 78 & \\
\hline Mortality rate, per 1,000 person-years & 13.0 & 13.0 & 17.5 & 20.7 & \\
\hline Unadjusted HR (95\% Cl) & 1 (Reference) & $1.00(0.69-1.46)$ & $1.36(0.95-1.94)$ & $1.61(1.14-2.29)$ & 0.0016 \\
\hline Person-years of follow-up & 4,073 & 4,210 & 4,083 & 3,771 & \\
\hline \multicolumn{6}{|l|}{ All-cause mortality } \\
\hline No. with events & 188 & 204 & 229 & 278 & \\
\hline Mortality rate, per 1,000 person-years & 46.2 & 48.5 & 56.1 & 73.7 & \\
\hline Unadjusted HR (95\% Cl) & 1 (Reference) & $1.05(0.86-1.28)$ & $1.22(1.01-1.48)$ & $1.64(1.36-1.97)$ & $<0.0001$ \\
\hline Adjusted HR $(95 \% \mathrm{Cl})^{\mathrm{a}}$ & 1 (Reference) & $0.94(0.77-1.15)$ & $1.01(0.82-1.24)$ & $1.13(0.92-1.39)$ & 0.0909 \\
\hline Adjusted HR $(95 \% \mathrm{Cl})^{\mathrm{a}}$ of per 1-unit increase of log-transformed folate & \multicolumn{4}{|c|}{$1.16(0.99-1.36)$} & 0.0670 \\
\hline \multicolumn{6}{|l|}{ Cardiovascular mortality } \\
\hline No. with events & 50 & 59 & 61 & 87 & \\
\hline Mortality rate, per 1,000 person-years & 12.3 & 14.0 & 14.9 & 23.1 & \\
\hline Unadjusted HR (95\% Cl) & 1 (Reference) & $1.14(0.78-1.66)$ & $1.22(0.84-1.77)$ & $1.91(1.35-2.70)$ & $<0.0001$ \\
\hline
\end{tabular}

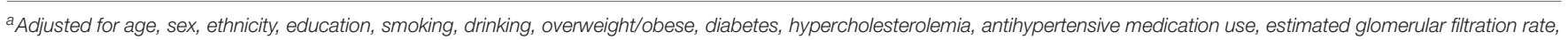
serum vitamin $B_{12}$, and $C$-reactive protein. HR, hazard ratio; $\mathrm{Cl}$, confidence interval.

homocysteine accumulates, leading to arterial calcification and atherosclerosis. High levels of RBC folate were associated with a high risk of severe abdominal aortic calcification, which might be evidence for this theory (11). In contrast, we did not find a significant association between serum folate and mortality among hypertensive patients with elevated homocysteine, which suggests that serum and RBC folate play different roles in the etiology of certain diseases. Routinely monitoring of $\mathrm{RBC}$ folate is recommended for folic acid supplement users.

This study has several limitations. First, although the NHANES population is a nationally representative sample of US adults, we limited the sample of this study to hypertensive patients, which may impair the representativeness. Second, limited by the small sample size, we only included CVDrelated mortality but not other cause-specific mortality. Third, due to the lack of data on folic acid supplement use, we were unable to further differentiate the associations between RBC folate and mortality by folic acid supplement use history. Fourth, folate status was investigated only once at baseline.
Patients' lifestyle habits including diet and tobacco smoking likely changes after diagnosis of H-type hypertension. Such changes could have an impact on long-term outcome. Unfortunately, this study did not collect these mentioned variables during the follow-up, and this limitation needs to be improved in future studies.

\section{CONCLUSIONS}

In conclusion, findings from our study suggest that a high level of $\mathrm{RBC}$ folate is associated with an increased risk of cardiovascular mortality among hypertensive patients with elevated homocysteine. Further studies on larger samples are warranted to confirm our findings.

\section{DATA AVAILABILITY STATEMENT}

The raw data supporting the conclusions of this article will be made available by the authors, without undue reservation. 


\section{ETHICS STATEMENT}

The studies involving human participants were reviewed and approved by National Center for Health Statistics (NCHS). The patients/participants provided their written informed consent to participate in this study.

\section{AUTHOR CONTRIBUTIONS}

LZhou designed the present study and performed data analysis. LZhou and $\mathrm{HH}$ contributed equally to the writing of this article. XW, LZhao, ML, JT, and GL critically revised and edited the manuscript for important intellectual content. YC, JL, FC, and GL contributed to the data interpretation. All

\section{REFERENCES}

1. GBD 2017 Causes of Death Collaborators. Global, regional, and national agesex-specific mortality for 282 causes of death in 195 countries and territories, 1980-2017: a systematic analysis for the global burden of disease study 2017. Lancet. (2018) 392:1736-88. doi: 10.1016/S0140-6736(18)32203-7

2. Mills KT, Stefanescu A, He J. The global epidemiology of hypertension. Nat Rev Nephrol. (2020) 16:223-37. doi: 10.1038/s41581-019-0244-2

3. NCD Risk Factor Collaboration (NCD-RisC). Worldwide trends in hypertension prevalence and progress in treatment and control from 1990 to 2019: a pooled analysis of 1201 populationrepresentative studies with 104 million participants. Lancet. (2021) 398:957-80. doi: 10.1016/S0140-6736(21)01330-1

4. Wald DS, Law M, Morris JK. Homocysteine and cardiovascular disease: evidence on causality from a meta-analysis. BMJ. (2002) 325:1202. doi: 10.1136/bmj.325.7374.1202

5. Zhao W, Gao F, Lv L, Chen X. The interaction of hypertension and homocysteine increases the risk of mortality among middle-aged and older population in the United States. J Hypertens. (2021) 40:254-63. doi: 10.1097/HJH.0000000000003002

6. Towfighi A, Markovic D, Ovbiagele B. Pronounced association of elevated serum homocysteine with stroke in subgroups of individuals: a nationwide study. J Neurol Sci. (2010) 298:153-7. doi: 10.1016/j.jns.2010.07.013

7. Qin X, Huo Y. H-Type hypertension, stroke and diabetes in China: opportunities for primary prevention. J Diabetes. (2016) 8:38-40. doi: 10.1111/1753-0407.12333

8. Carmel R. Folic acid. In: Shils M, Shike M, Ross A, Caballero B, Cousins RJ, editors. Modern Nutrition in Health and Disease. 11th ed. Baltimore, MD: Lippincott Williams \& Wilkins (2005). p. 470-81.

9. Selhub J, Jacques PF, Wilson PW, Rush D, Rosenberg IH. Vitamin status and intake as primary determinants of homocysteinemia in an elderly population. JAMA. (1993) 270:2693-8. doi: 10.1001/jama.1993.035102200 49033

10. Huo Y, Li J, Qin X, Huang Y, Wang X, Gottesman RF, et al. Efficacy of folic acid therapy in primary prevention of stroke among adults with hypertension in China: the CSPPT randomized clinical trial. JAMA. (2015) 313:132535. doi: 10.1001/jama.2015.2274

11. Zhou L, Wen X, Peng Y, Guo M, Zhao L. Red blood cell folate and severe abdominal aortic calcification: results from the NHANES 2013-2014. Nutr Metab Cardiovasc Dis. (2021) 31:186-92. doi: 10.1016/j.numecd.2020.08.020

12. Chen MY, Rose CE, Qi YP, Williams JL, Yeung LF, Berry RJ, et al. Defining the plasma folate concentration associated with the red blood cell folate concentration threshold for optimal neural tube defects prevention: a population-based, randomized trial of folic acid supplementation. Am J Clin Nutr. (2019) 109:1452-61. doi: 10.1093/ajcn/nqz027

13. Zipf G, Chiappa M, Porter KS, Ostchega Y, Lewis BG, Dostal J. National health and nutrition examination survey: plan and operations, 1999-2010. Vital Health Stat. (2013) 56:1-37. authors approved the final version of the manuscript to be published.

\section{FUNDING}

This work was supported by the Scientific Research Starting Funding for Young Scholars in Sichuan Provincial People's Hospital. The funding source had no role in the design, analysis, or writing of this manuscript.

\section{ACKNOWLEDGMENTS}

We truly appreciate all the NHANES participants and staff for their invaluable efforts and contributions.

14. WHO expert Consultation. Appropriate body-mass index for Asian populations and its implications for policy and intervention strategies. Lancet. (2004) 363:157-63. doi: 10.1016/S0140-6736(03)15268-3

15. Levey AS, Stevens LA, Schmid CH, Zhang Y, Castro III AF, Feldman HI, et al. A new equation to estimate glomerular filtration rate. Ann Intern Med. (2009) 150:604-12. doi: 10.7326/0003-4819-150-9-200905050-00006

16. US Centers for Disease Control and Prevention. 2015 Public-Use Linked Mortality File. 56:1-37. Available online at: https://www.cdc.gov/nchs/datalinkage/mortality-public.htm (accessed September 10, 2021).

17. Sonawane K, Zhu Y, Chan W, Aguilar D, Deshmukh AA, SuarezAlmazor ME. Association of serum folate levels with cardiovascular mortality among adults with rheumatoid arthritis. JAMA Netw Open. (2020) 3:e200100. doi: 10.1001/jamanetworkopen.2020.0100

18. Loria CM, Ingram DD, Feldman JJ, Wright JD, Madans JH. Serum folate and cardiovascular disease mortality among US men and women. Arch Intern Med. (2000) 160:3258-62. doi: 10.1001/archinte.160.21.3258

19. Dangour AD, Breeze E, Clarke R, Shetty PS, Uauy R, Fletcher AE. Plasma homocysteine, but not folate or vitamin B-12, predicts mortality in older people in the United Kingdom. J Nutr. (2008) 138:11218. doi: $10.1093 /$ jn/138.6.1121

20. Nkemjika S, Ifebi E, Cowan LT, Chun-Hai Fung I, Twum F, Liu $\mathrm{F}$, et al. Association between serum folate and cardiovascular deaths among adults with hypertension. Eur J Clin Nutr. (2020) 74:9708. doi: 10.1038/s41430-019-0533-7

21. Twum F, Morte N, Wei Y, Nkemjika S, Liu F, Zhang J. Red blood cell folate and cardiovascular deaths among hypertensive adults, an 18year follow-up of a national cohort. Hypertens Res. (2020) 43:93847. doi: 10.1038/s41440-020-0482-5

22. Liu M, Zhang Z, Zhou C, Li Q, He P, Zhang Y, et al. Relationship of several serum folate forms with the risk of mortality: a prospective cohort study. Clin Nutr. (2021) 40:4255-62. doi: 10.1016/j.clnu.2021.01.025

23. Yan LJ, Zhang FR, Zeng YR, Zheng Y. Serum folate and all-cause mortality is of non-linear relationship among population with chronic kidney disease: a retrospective cohort study. Int J Gen Med. (2021) 14:2695702. doi: 10.2147/IJGM.S314904

24. Kyte B, Ifebi E, Shrestha S, Charles S, Liu F, Zhang J. High red blood cell folate is associated with an increased risk of death among adults with diabetes, a 15-year follow-up of a national cohort. Nutr Metab Cardiovasc Dis. (2015) 25:997-1006. doi: 10.1016/j.numecd.2015.08.007

25. Cui $\mathrm{R}$, Iso $\mathrm{H}$, Date $\mathrm{C}$, Kikuchi $\mathrm{S}$, Tamakoshi A. Dietary folate and vitamin b6 and B12 intake in relation to mortality from cardiovascular diseases: Japan collaborative cohort study. Stroke. (2010) 41:1285-9. doi: 10.1161/STROKEAHA.110.578906

26. Chen S, Honda T, Hata J, Sakata S, Furuta Y, Yoshida D, et al. High serum folate concentrations are associated with decreased risk of mortality among japanese adults. J Nutr. (2021) 151:657-65. doi: 10.1093/jn/nxaa382

27. Gopinath B, Flood VM, Rochtchina E, Thiagalingam A, Mitchell P. Serum homocysteine and folate but not vitamin B12 are predictors of 
CHD mortality in older adults. Eur J Prev Cardiol. (2012) 19:14209. doi: $10.1177 / 1741826711424568$

28. Peng Y, Dong B, Wang Z. Serum folate concentrations and all-cause, cardiovascular disease and cancer mortality: a cohort study based on 19992010 national health and nutrition examination survey (NHANES). Int $J$ Cardiol. (2016) 219:136-42. doi: 10.1016/j.ijcard.2016.06.024

29. Antoniades C, Antonopoulos AS, Tousoulis D, Marinou K, Stefanadis C. Homocysteine and coronary atherosclerosis: from folate fortification to the recent clinical trials. Eur Heart J. (2009) 30:6-15. doi: 10.1093/eurheartj/ehn515

30. Aghamohammadi V, Gargari BP, Aliasgharzadeh A. Effect of folic acid supplementation on homocysteine, serum total antioxidant capacity, and malondialdehyde in patients with type 2 diabetes mellitus. J Am Coll Nutr. (2011) 30:210-5. doi: 10.1080/07315724.2011.10719962

31. Van Campenhout A, Moran CS, Parr A, Clancy P, Rush C, Jakubowski H, et al. Role of homocysteine in aortic calcification and osteogenic cell differentiation. Atherosclerosis. (2009) 202:557-66. doi: 10.1016/j.atherosclerosis.2008.05.031

32. Selhub J, Morris MS, Jacques PF. In vitamin B12 deficiency, higher serum folate is associated with increased total homocysteine and methylmalonic acid concentrations. Proc Natl Acad Sci USA. (2007) 104:19995-20000. doi: 10.1073/pnas.0709487104
Conflict of Interest: The authors declare that the research was conducted in the absence of any commercial or financial relationships that could be construed as a potential conflict of interest.

Publisher's Note: All claims expressed in this article are solely those of the authors and do not necessarily represent those of their affiliated organizations, or those of the publisher, the editors and the reviewers. Any product that may be evaluated in this article, or claim that may be made by its manufacturer, is not guaranteed or endorsed by the publisher.

Copyright (c) 2022 Zhou, Huang, Wen, Chen, Liao, Chen, Zhao, Liu, Tao and Li. This is an open-access article distributed under the terms of the Creative Commons Attribution License (CC BY). The use, distribution or reproduction in other forums is permitted, provided the original author(s) and the copyright owner(s) are credited and that the original publication in this journal is cited, in accordance with accepted academic practice. No use, distribution or reproduction is permitted which does not comply with these terms. 